\title{
Low Serological Prevalence of SARS-CoV-2 Antibodies in Cancer Patients at a German University Oncology Center
}

\author{
Oliver Overheu $^{\mathrm{a}}$ Daniel R. Quast ${ }^{\mathrm{b}}$ Wolfgang E. Schmidt ${ }^{\mathrm{b}}$ \\ Türkan Sakinç-Güler ${ }^{c}$ Anke Reinacher-Schick ${ }^{a}$ \\ aDepartment of Hematology and Oncology with Palliative Care, St. Josef-Hospital, Ruhr-University Bochum, \\ Bochum, Germany; ${ }^{b}$ Department of Internal Medicine, St. Josef-Hospital, Ruhr-University Bochum, Bochum, \\ Germany; ' Central Laboratory, St. Josef-Hospital, Ruhr-University Bochum, Bochum, Germany
}

\section{Keywords}

Coronavirus disease-19 seroprevalence $\cdot$ Severe acute respiratory syndrome coronavirus type 2 - Antibody testing · Epidemiology · German oncology center

\footnotetext{
Abstract

Background: Coronavirus disease 2019 (COVID-19) cases in Germany, as in most other places in Europe or worldwide, are still highly prevalent. Vaccination rates currently remain low, putting cancer patients at a continued risk of infection with SARS-CoV-2, while prevalence of SARS-CoV-2 antibodies among cancer patients in Germany remains essentially unknown. Methods: Between August 2020 and February 2021, patients admitted to our hospital were prospectively enrolled in our COVID-19 biobank. Collected sera were analyzed for SARS-CoV-2-lgM/lgG using Elecsys Anti-SARSCoV-2 assay. Results: One hundred and ten patients with cancer were included in this study. With 71 (65\%) patients, most had active cancer treatment, mainly chemotherapy (56\%). The most frequent diagnosis was gastrointestinal cancer (54\%) with pancreatic cancer being the most common cancer type (24\%). Hematologic malignancies were present in 21 patients (17\%). Among the cancer patients first diagnosed during the pandemic, the rate of palliative treatment situations tended to be higher (76\% vs. $67 \%, p=0.17$ ). A history of SARS-CoV-2 infection was documented in 15 (14\%) patients; however, SARS-CoV-2 antibodies were de-
}

tected in 10 (67\%) patients only. Of the patients without a history of SARS-CoV-2 infection, none displayed SARS-CoV-2 antibodies. Conclusion: In the present single-center experience, a low serological prevalence of SARS-CoV-2 antibodies among cancer patients even after SARS-CoV-2 infection was found. The results support continued strict preventive measures as well as efforts toward faster vaccination, due to a low immunity level in the population.

๑) 2021 S. Karger AG, Basel

\section{Introduction}

The coronavirus disease 2019 (COVID-19) pandemic caused by the severe acute respiratory syndrome coronavirus type 2 (SARS-CoV-2) remains a worldwide public health concern $[1,2]$. Previous studies suggested presence of a cancer diagnosis to be a risk factor for a more serious course of COVID-19 [3]. Especially patients with a pulmonary manifestation or a hemato-oncological disease appeared to be at a higher risk for severe COVID-19 disease $[4,5]$. A study by Williamson et al. [6] lately found that an increased risk for COVID-19-related death is associated with a recent cancer diagnosis. However, the cohort of cancer patients is heterogeneous, and data on the rate of asymptomatic carriers among these patients are scarce. Additionally, risk of nosocomial transmission of COVID-19 is a serious concern for patients that rely on regular in- or outpatient visits. While rigorous infection 
control measures help prevent nosocomial infection [7], data on their effectiveness for the above-mentioned collective in Germany are limited.

SARS-CoV-2 PCR testing following a nasopharyngeal swab is the current standard for detection of SARS-CoV-2 infection [8]. SARS-CoV-2 IgG and/or IgM antibody testing may complement PCR testing [9]. Only half of the patients show positive results of SARS-CoV-2 IgG and IgM antibodies within the first week, and seroconversion usually occurs 2 weeks after symptom onset [10-12]. Thus, antibody testing is not essential for the acute detection of SARS-CoV-2 infection, but may be utilized in detecting previous and potentially asymptomatic infections as well as subsequent immunity following a SARS-CoV-2 infection $[13,14]$.

Data concerning the prevalence of SARS-CoV-2 antibodies are ambiguous and highly dependent from the local pandemic status [15]. Thus, while an Austrian study reported a prevalence of $3.6 \%$ among oncological patients [16], prevalence among outpatient cancer patients in high prevalence areas like Madrid (Spain) was reported to be around 30\% [17]. However, these results represent the status quo after the pandemic's "first wave" and do not respect the worldwide surge in SARS-CoV-2 infections in fall/winter of 2020/21 [18]. Therefore, the present study aims to report the current prevalence of SARS-CoV-2 antibodies among cancer patients in a German university hospital after 1 year of COVID-19 pandemic.

\section{Methods}

Patients in this study were prospectively enrolled into our COVID-19 biobanking study between August 2020 and February 2021. To be eligible for enrollment, patients had to have suspected or proven infection with SARS-CoV-2 and/or belonged to 1 or more specified risk groups, including cancer patients. This study incorporated a subanalysis of all registered patients with an oncological or hemato-oncological diagnosis, who presented at our department.

According to the study protocol of our COVID-19 biobank, patients had their blood drawn and medical data documented at time of enrollment and/or specified dates afterward during clinical routine. Written informed consent was obtained from all included patients. Blood serum samples were centrifuged and stored locally at $-80^{\circ} \mathrm{C}$. The study was approved by the Ethics Committee of the Medical Faculty, Ruhr-University Bochum (Approval No. 206953-bio).

All laboratory analyses were performed at the Central Laboratory of St. Josef-Hospital Bochum, Hospital of the Ruhr-University Bochum. SARS-CoV-2-IgM/IgG were analyzed using the Elecsys Anti-SARS-CoV-2 assay. This test utilizes a recombinant protein representing the nucleocapsid $(\mathrm{N})$-antigen for qualitative detection of high-affinity antibodies against SARS-CoV-2. Results are given as a numeric cutoff index (COI) with results $\geq 1.0$ representing reactivity and positivity, respectively, for SARS-CoV-2 antibodies. This assay proved to be highly sensitive with a sensitivity up to $99.5 \%>14$ days after positive PCR testing for SARS-CoV-2 infection. From day $0-6$, it has a sensitivity of $60.2 \%$ and from day
7-13 85.3\%. All tests were performed according to the manufacturer's manual.

Descriptive data are presented as $n(\%)$ or median (range of values). All own percentual results are rounded to the nearest full number. Data were analyzed using Fisher's exact test or Student's $t$ test. Results were considered significant at $\alpha=0.05$.

\section{Results}

\section{Patient Characteristics}

One hundred and ten patients with a total of 123 diagnosed cancers were included in this study. Baseline characteristics are presented in Table 1. Forty-six percent of the patients were female, and median age was 67.5 years. Most patients (65\%) were undergoing active cancer treatment. The most frequent diagnosis was gastrointestinal cancer in general (54\%) with pancreatic cancer being the most common cancer type (24\%). Hemato-oncological diseases (leukemia, lymphoma, myeloma, and myelodysplastic syndrome) accounted for $17 \%$ and lung cancer for $8 \%$ of the cases. Median time since initial diagnosis was 3 months (0-192 months).

Most patients were treated with chemotherapy (62, $56 \%)$ and about a third $(35,32 \%)$ received immunotherapy $(10,9 \%)$ or targeted therapy $(25,23 \%)$. Only one of these patients was solely treated with immunotherapy, whereas the others got combinations with either chemotherapy (3), chemotherapy and radiation (5), or a targeted therapy (1). Among those on a targeted therapy, 18 patients received additional chemotherapy and 1 the forementioned immunotherapy. Nineteen (12\%) patients were in follow-up, while 7 (4\%) received best supportive care. Eighty-five (69\%) treatments were palliative and 39 (32\%) were curatively intended. Fifty-four percent of the patients received inpatient treatment or diagnostics. Twenty-four patients (22\%) did not receive any active cancer treatment because they presented with the initial diagnosis of a malignant disease. The rate of palliative treatment situations among those first diagnosed during the pandemic was higher than in the whole population (76\% vs. $67 \%$ ), although not statistically significant $(p=0.17)$. Sixty-nine patients $(63 \%)$ suffered from metastatic disease (if applicable) and 26 had pulmonary metastasis (24\%).

\section{SARS-CoV-2 Antibody Prevalence}

Fifteen patients had a positive history of SARS-CoV-2 infection (14\%, see Table 2). Positive results for SARS$\mathrm{CoV}-2$ antibodies were detected in 10 of these patients (66\%), and the median cutoff index (COI) was 113.5. There was no statistically significant difference in COI between patients on antitumor treatment and pretreatment $(p=0.33)$. Five patients with a history of SARS$\mathrm{CoV}-2$ infection had a negative test for SARS-CoV-2 an- 
Table 1. Patient characteristics

\begin{tabular}{|c|c|}
\hline Median age, years & $67.5(18-88)$ \\
\hline \multicolumn{2}{|l|}{ Sex, $n(\%)$} \\
\hline Female & $51(46)$ \\
\hline Male & $59(54)$ \\
\hline \multicolumn{2}{|l|}{ Cancer type, $n(\%)$} \\
\hline Colorectal & $17(14)$ \\
\hline Pancreas & $29(24)$ \\
\hline Esophageal & $7(6)$ \\
\hline Gastric & $1(1)$ \\
\hline Anal & $3(2)$ \\
\hline Hepatobiliary & $7(6)$ \\
\hline Lung & $10(8)$ \\
\hline Lymphoma & $7(6)$ \\
\hline Leukemia & $8(7)$ \\
\hline Myeloma & $5(4)$ \\
\hline Urogenital & $5(4)$ \\
\hline Gynecological & $1(1)$ \\
\hline Breast & $7(6)$ \\
\hline Prostate & $1(1)$ \\
\hline Others & $15(12)$ \\
\hline \multicolumn{2}{|l|}{ Intent of treatment, $n$ (\%) } \\
\hline Curative & $39(32)$ \\
\hline Palliative & $84(68)$ \\
\hline Outpatient & $56(46)$ \\
\hline Inpatient & $67(54)$ \\
\hline UICC I* (*if applicable) & $8(7)$ \\
\hline ॥ & $8(7)$ \\
\hline III & $14(13)$ \\
\hline IV & $69(63)$ \\
\hline M1 (PUL) & $26(24)$ \\
\hline Patients on active cancer treatment, $n$ (\%) & $71(65)$ \\
\hline Chemotherapy & $62(56)$ \\
\hline Radiation & $8(7)$ \\
\hline Immunotherapy & $10(9)$ \\
\hline Targeted therapy & $25(23)$ \\
\hline Hormonal therapy & $2(2)$ \\
\hline Patients without active treatment, $n$ (\%) & $39(36)$ \\
\hline Best supportive care & $7(6)$ \\
\hline Aftercare & $8(7)$ \\
\hline Initial diagnosis/pretreatment & $24(22)$ \\
\hline Median time since initial diagnosis, months & $3(0-192)$ \\
\hline
\end{tabular}

Data are presented as $n$ (\%) or median (range of values).

tibodies. None of them had a relevant comorbidity that would lead to an impaired immune response. Characteristics of all patients with a history of SARS-CoV-2 infection are presented in Table 3. None of the patients without a known history of SARS-CoV-2 infection were tested positive for SARS-CoV-2 antibodies, exhibiting a nonexistent serological prevalence of SARS-CoV-2 antibodies among the majority of our collective. SARS-CoV-2 infection of $8(54 \%)$ patients occurred nosocomial.

It is worth noting that except one, those patients with a history of SARS-CoV-2 infection and negative serological testing had their blood drawn within up to 2 weeks after their first positive PCR testing (2-10 days), where antibody response is usually still low and the sensitivity
Table 2. Results of SARS-CoV-2 antigen testing

$\begin{array}{lll}\text { Patients with history of COVID-19 } & 15 & 13.6 \% \\ \text { Positive SARS-CoV-2 IgG/IgM } & 10 & 9 \% \\ \text { Negative SARS-CoV-2 IgG/lgM } & 100 & 91 \% \\ \text { Median SARS-CoV-2 COI } & 113.5 & (17.9-187)\end{array}$

Data are presented as $n$ (\%) or median (range of values). COI, cutoff index.

of the test assay is limited $[11,19]$. Due to various reasons, including early discharge because of mild or asymptomatic disease, no follow-up samples were possible. The remaining patient (\#10) had mild symptoms and a positive PCR test in October 2020 before his diagnosis with pancreatic cancer. The possibility of a false-positive PCR test should be taken into consideration in this case.

None of our cancer patients who suffered from COVID-19 had a lethal course of the infection or required any kind of mechanical ventilation. Nonetheless, 4 patients $(27 \%)$ had a severe course, requiring prolonged hospitalization and intensified treatment with oxygen. The minority of our patients with a nosocomial infection had a severe course (38\%). Additionally, none of the patients reported signs of a long COVID syndrome, yet.

\section{Discussion}

COVID-19 cases in Germany, as in most other places in Europe or worldwide, are still highly prevalent [18], although incidence rates recently declined slowly in May 2021. Vaccination rates currently remain low, putting oncological patients at a continued risk of infection with SARS-CoV-2. Our results with a low serological prevalence of SARS-CoV-2 antibodies among cancer patients even after SARS-CoV-2 infection and no prevalence among those without a history of COVID-19 support continued strict preventive measures as well as efforts toward faster vaccination, due to low immunity in the population.

At the time of initiation of this study, serological prevalence among asymptomatic ambulatory patients in the North Rhine-Westphalia region in Germany was reported to be $1.2 \%$ [20]. A nearby tertiary hospital reported an incidence of $1.6 \%$ among health care workers [21]. This is consistent with our findings among asymptomatic patients, as well as with 2 studies from Austria that reported COVID-19 prevalence among cancer patients of $0.4 \%$ and $2.4 \%$, respectively, and a French study describing a seroprevalence of SARS-CoV-2 antibodies of $1.7 \%$ in a cohort of oncological patients [16, 22, 23]. From the original pandemic epicenter in Wuhan, an incidence of $2.9 \%$ of asymptomatic infections among cancer patients was 
reported [24]. A large, prospective Belgian study came to similar results [25]. However, the seroprevalence in our cohort (9\%) is still higher than the forementioned numbers. This might be due to selection bias, as this is an analysis of a subgroup of patients included in our COVID-19 biobank. As such, patients with an active or known history of COVID-19 were preferably included. Nonetheless, these results are still lower than those in regions with severe outbreaks of COVID-19, like a study reporting a prevalence of 30\% among cancer outpatients in Madrid [17]. The German Robert Koch-Institute estimated the seroprevalence of COVID-19 in Germany at $7.8 \%$ in March 2021.

The ambiguous results of antibody testing among patients with an active infection with SARS-CoV-2 highlight a demand for longitudinal testing to avoid false-negative results. A COI below median in these patients might be due to hematological disease (\#4,\#5), antigen test shortly after COVID-19 diagnosis (\#2), long time since SARS-CoV-2 infection (\#14), or immunosenescence (\#15). This also underlines the fact that antibody tests should not be used for frontline detection of an active SARS-CoV-2 infection due to low sensitivity $[26,27]$. PCR testing remains the gold standard in this case. However, regular measurement of antibody titers might also prove useful among cancer patients who have already received their COVID-19 vaccination to identify nonresponders or patients with declining antibody levels. Patients with an increased risk of low or no response to a SARS-CoV-2 vaccination naturally include such with a hematological disease or on certain types of treatment (e.g., anti-CD20 antibodies and CAR T-cell therapy) [28-32]. While durability of immune response in general remains high, Eliakim-Raz et al. [33] already reported a decline of antibody titers in cancer patients after 4 months. Seropositivity has been shown to be a useful correlate with protection from infection [34], although the degree of contribution of cellular immunity toward a durable immune response remains unclear and requires further evaluation [35].

None of our patients with COVID-19 had a lethal course, and severe courses were scarce even among those with a nosocomial infection. While previous data suggested a lower mortality among nosocomial-acquired SARS-CoV-2 infections in general [36], an increased mortality among cancer patients with hospital-acquired COVID-19 has been reported by Elkrief et al. [37].

Given the association of increased risk of mortality due to COVID-19 and a cancer diagnosis, especially for lung cancer and hematological malignancies [6,38-40], as well as scarce resources in the context of the pandemic, various recommendations regarding the management of cancer care were issued last year [41-43]. Continuation of cancer treatment was generally recommended [5, 44-46]. Additionally, the COVID-19 pandemic led to a signifi-

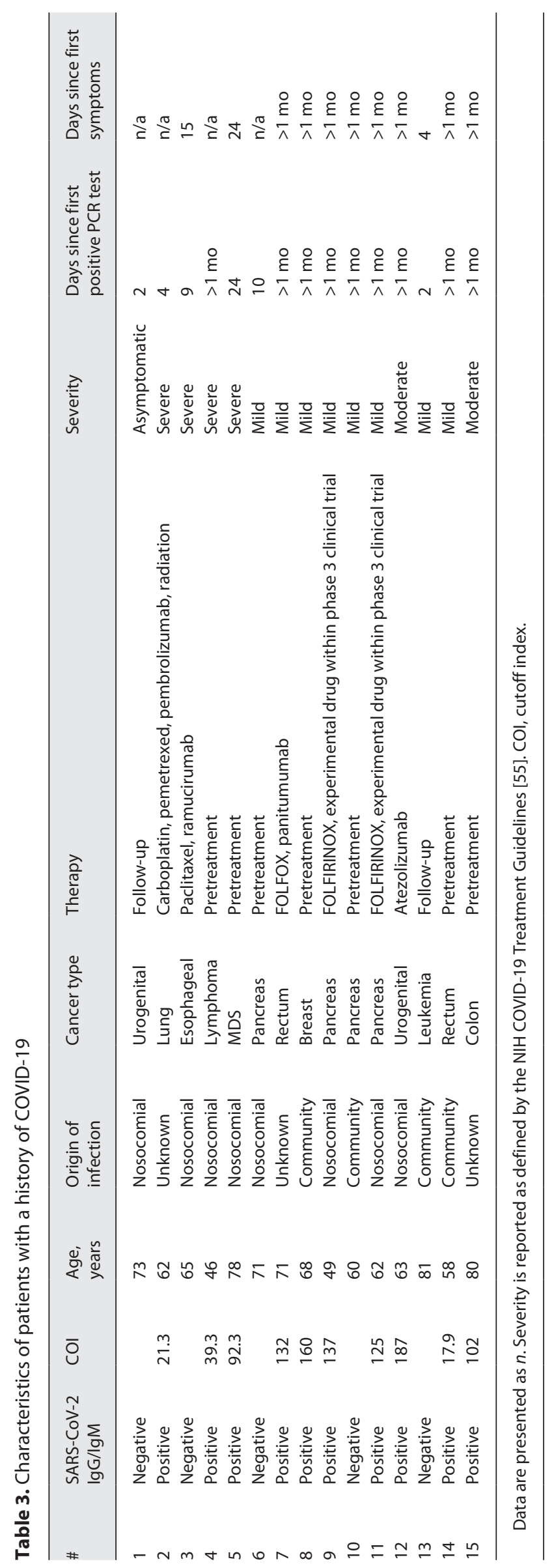

Oncol Res Treat 2022;45:112-117

DOI: $10.1159 / 000520572$ 
cant decrease in cancer screenings [47-49]. A subsequent increase in cancer-related deaths due to delayed diagnosis is expected [50]. In this context, our data suggest that oncological diagnostics as well as treatments can and should safely be continued during a pandemic, when and while strict safety measures are in place.

As our data suggest a generally low immunity against SARS-CoV-2 among oncological patients in Germany, vaccination remains highly recommended [51, 52]. Especially as SARS-CoV-2 vaccine appears to be a scarce resource, the present results highlight the need for further studies to identify whether there are groups of patients that do not benefit from vaccination (e.g., patients with a B-cell depletion or severe combined B-/T-cell impairment or deficiencies) [53, 54].

\section{Statement of Ethics}

This study protocol was reviewed and approved by the Ethics Committee of the Medical Faculty, Ruhr-University Bochum, Approval No. 20-6953-bio, and the study was conducted in accordance with the Declaration of Helsinki. Written informed consent was obtained from all participants prior to inclusion.

\section{Conflict of Interest Statement}

The authors declare no conflicts of interest.

\section{Funding Sources}

No external source of funding was present.

\section{Author Contributions}

O.O. collected and analyzed the data and drafted and revised the manuscript. T. S.-G. was responsible for the laboratory analyses. All authors contributed to the conceptional design of the study. All authors read and approved the final manuscript.

\section{Data Availability Statement}

The data used and analyzed during this study are available from the corresponding author upon request.

\section{References}

1 Wu F, Zhao S, Yu B, Chen YM, Wang W, Song ZG, et al. A new coronavirus associated with human respiratory disease in China. $\mathrm{Na}-$ ture. 2020;579(7798):265-9.

2 Ioannidis JPA. Global perspective of COVID-19 epidemiology for a full-cycle pandemic. Eur J Clin Invest. 2020;50(12):e13423.

3 Rüthrich MM, Giessen-Jung C, Borgmann S, Classen AY, Dolff S, Grüner B, et al. COVID-19 in cancer patients: clinical characteristics and outcome-an analysis of the LEOSS registry. Ann Hematol. 2021;100(2):383-93.

4 Dai M, Liu D, Liu M, Zhou F, Li G, Chen Z, et al. Patients with cancer appear more vulnerable to SARS-COV-2: a multi-center study during the COVID-19 outbreak. Cancer Discov. 2020.

5 Lee LYW, Cazier JB, Starkey T, Briggs SEW, Arnold R, Bisht V, et al. COVID-19 prevalence and mortality in patients with cancer and the effect of primary tumour subtype and patient demographics: a prospective cohort study. Lancet Oncol. 2020;21(10):1309-16.

6 Williamson EJ, Walker AJ, Bhaskaran K, Bacon S, Bates C, Morton CE, et al. Factors associated with COVID-19-related death using Open safely. Nature. 2020;584(7821):430-6.

7 Rhee C, Baker M, Vaidya V, Tucker R, Resnick A, Morris CA, et al. Incidence of nosocomial COVID-19 in patients hospitalized at a large US academic medical center. JAMA Netw Open. 2020;3(9):e2020498.

8 D'Cruz RJ, Currier AW, Sampson VB. Laboratory testing methods for novel severe acute respiratory syndrome-coronavirus-2 (SARSCoV-2). Front Cell Dev Biol. 2020;8:468.
9 Zhao J, Yuan Q, Wang H, Liu W, Liao X, Su $\mathrm{Y}$, et al. Antibody responses to SARS-CoV-2 in patients with novel coronavirus disease 2019. Clin Infect Dis. 2020;71(16):2027-34.

10 Wölfel R, Corman VM, Guggemos W, Seilmaier M, Zange S, Müller MA, et al. Virological assessment of hospitalized patients with COVID-2019. Nature. 2020;581(7809):4659.

11 Qu J, Wu C, Li X, Zhang G, Jiang Z, Zhu Q, et al. Profile of Immunoglobulin $\mathrm{G}$ and IgM antibodies against severe acute respiratory syndrome coronavirus 2 (SARS-CoV-2). Clin Infect Dis. 2020;71(16):2255-8.

12 Sun B, Feng Y, Mo X, Zheng P, Wang Q, Li P, et al. Kinetics of SARS-CoV-2 specific IgM and $\operatorname{IgG}$ responses in COVID-19 patients. Emerg Microbes Infect. 2020;9(1):940-8.

13 Hoffman T, Nissen K, Krambrich J, Rönnberg B, Akaberi D, Esmaeilzadeh M, et al. Evaluation of a COVID-19 IgM and IgG rapid test; an efficient tool for assessment of past exposure to SARS-CoV-2. Infect Ecol Epidemiol. 2020;10(1):1754538.

14 Li Z, Yi Y, Luo X, Xiong N, Liu Y, Li S, et al. Development and clinical application of a rapid IgM-IgG combined antibody test for SARS-CoV-2 infection diagnosis. J Med Virol. 2020;92(9):1518-24.

15 Rostami A, Sepidarkish M, Leeflang MMG, Riahi SM, Nourollahpour Shiadeh M, Esfandyari S, et al. SARS-CoV-2 seroprevalence worldwide: a systematic review and metaanalysis. Clin Microbiol Infect. 2021;27(3): $331-40$.
16 Fuereder T, Berghoff AS, Heller G, Haslacher $\mathrm{H}$, Perkmann T, Strassl R, et al. SARS-CoV-2 seroprevalence in oncology healthcare professionals and patients with cancer at a tertiary care centre during the COVID-19 pandemic. ESMO Open. 2020;5(5):e000889.

17 Cabezón-Gutiérrez L, Custodio-Cabello S, Palka-Kotlowska M, Oliveros-Acebes E, García-Navarro MJ, Khosravi-Shahi P. Seroprevalence of SARS-CoV-2-specific antibodies in cancer outpatients in Madrid (Spain): a single center, prospective, cohort study and a review of available data. Cancer Treat Rev. 2020;90: 102102.

18 Post L, Culler K, Moss CB, Murphy RL, Achenbach CJ, Ison MG, et al. European SARS-CoV-2 surveillance: longitudinal trend analyses of wave two. JMIR Public Health Surveill. 2021.

19 Egger M, Bundschuh C, Wiesinger K, Gabriel C, Clodi M, Mueller T, et al. Comparison of the Elecsys ${ }^{\oplus}$ Anti-SARS-CoV-2 immunoassay with the EDI ${ }^{\text {tw }}$ enzyme linked immunosorbent assays for the detection of SARS-CoV-2 antibodies in human plasma. Clin Chim Acta. 2020;509:18-21.

20 Herrmann BL. The prevalence rate of antiSARS-CoV-2-IgG is $1.2 \%$ : screening in asymptomatic outpatients in Germany (Northrhine-Westfalia). MMW Fortschr Med. 2020; 162(14):44-6.

21 Korth J, Wilde B, Dolff S, Anastasiou OE, Krawczyk A, Jahn M, et al. SARS-CoV-2-specific antibody detection in healthcare workers in Germany with direct contact to COVID-19 patients. J Clin Virol. 2020;128:104437. 
22 Berghoff AS, Gansterer M, Bathke AC, Trutschnig W, Hungerländer P, Berger JM, et al. SARS-CoV-2 testing in patients with cancer treated at a tertiary care hospital during the COVID-19 pandemic. J Clin Oncol. 2020; 38(30):3547-54.

23 Ladoire S, Goussot V, Redersdorff E, Cueff A, Ballot E, Truntzer C, et al. Seroprevalence of SARS-CoV-2 among the staff and patients of a French cancer centre after first lockdown: the canSEROcov study. Eur J Cancer. 2021; 148:359-70.

24 Yin P, Zeng R, Duan YR, Zhang Y, Kuang XN, Zhang HF, et al. An analysis of cancer patients with asymptomatic infection of SARS-CoV-2 in a cancer center in Wuhan, China. Ann Oncol. 2020;31(10):1420-2.

25 van Dam P, Huizing M, Roelant E, Hotterbeekx A, De Winter FHR, Kumar-Singh S, et al. Immunoglobin $\mathrm{G} /$ total antibody testing for SARS-CoV-2: a prospective cohort study of ambulatory patients and health care workers in two Belgian oncology units comparing three commercial tests. Eur J Cancer. 2021; 148:328-39.

26 Scohy A, Anantharajah A, Bodéus M, Kabamba-Mukadi B, Verroken A, Rodriguez-Villalobos $\mathrm{H}$. Low performance of rapid antigen detection test as frontline testing for COVID-19 diagnosis. J Clin Virol. 2020;129: 104455.

27 Boum Y, Fai KN, Nicolay B, Mboringong AB, Bebell LM, Ndifon M, et al. Performance and operational feasibility of antigen and antibody rapid diagnostic tests for COVID-19 in symptomatic and asymptomatic patients in Cameroon: a clinical, prospective, diagnostic accuracy study. Lancet Infect Dis. 2021.

28 Addeo A, Shah PK, Bordry N, Hudson RD, Albracht B, Di Marco M, et al. Immunogenicity of SARS-CoV-2 messenger RNA vaccines in patients with cancer. Cancer Cell. 2021; 39(8):1091-8.e2.

29 Thakkar A, Gonzalez-Lugo JD, Goradia N, Gali R, Shapiro LC, Pradhan K, et al. Seroconversion rates following COVID-19 vaccination among patients with cancer. Cancer Cell. 2021;39(8):1081-90.e2.

30 Van Oekelen O, Gleason CR, Agte S, Srivastava K, Beach KF, Aleman A, et al. Highly variable SARS-CoV-2 spike antibody responses to two doses of COVID-19 RNA vaccination in patients with multiple myeloma. Cancer Cell. 2021;39(8):1028-30.

31 Terpos E, Trougakos IP, Gavriatopoulou M, Papassotiriou I, Sklirou AD, Ntanasis-Stathopoulos I, et al. Low neutralizing antibody responses against SARS-CoV-2 in older patients with myeloma after the first BNT162b2 vaccine dose. Blood. 2021;137(26):3674-6.
32 Herishanu Y, Avivi I, Aharon A, Shefer G, Levi S, Bronstein Y, et al. Efficacy of the BNT162b2 mRNA COVID-19 vaccine in patients with chronic lymphocytic leukemia. Blood. 2021;137(23):3165-73.

33 Eliakim-Raz N, Massarweh A, Stemmer A, Stemmer SM. Durability of response to SARSCoV-2 BNT162b2 vaccination in patients on active anticancer treatment. JAMA Oncol. 2021.

34 Harvey RA, Rassen JA, Kabelac CA, Turenne W, Leonard S, Klesh R, et al. Association of SARS-CoV-2 seropositive antibody test with risk of future infection. JAMA Intern Med. 2021;181(5):672-9.

35 Zuo J, Dowell AC, Pearce H, Verma K, Long HM, Begum J, et al. Robust SARS-CoV-2-specific $\mathrm{T}$ cell immunity is maintained at 6 months following primary infection. Nat Immunol. 2021;22(5):620-6.

36 Carter B, Collins JT, Barlow-Pay F, Rickard F, Bruce E, Verduri A, et al. Nosocomial COVID-19 infection: examining the risk of mortality. The COPE-Nosocomial Study (COVID in older people). J Hosp Infect. 2020;106(2): 376-84.

37 Elkrief A, Desilets A, Papneja N, Cvetkovic L, Groleau C, Lakehal YA, et al. High mortality among hospital-acquired COVID-19 infection in patients with cancer: a multicentre observational cohort study. Eur J Cancer. 2020; 139:181-7.

38 de Joode K, Dumoulin DW, Tol J, Westgeest HM, Beerepoot LV, van den Berkmortel FWPJ, et al. Dutch oncology COVID-19 consortium: outcome of COVID-19 in patients with cancer in a nationwide cohort study. Eur J Cancer. 2020;141:171-84.

39 Liu H, Yang D, Chen X, Sun Z, Zou Y, Chen $\mathrm{C}$, et al. The effect of anticancer treatment on cancer patients with COVID-19: a systematic review and meta-analysis. Cancer Med. 2021; 10(3):1043-56.

40 Yang F, Shi S, Zhu J, Shi J, Dai K, Chen X. Clinical characteristics and outcomes of cancer patients with COVID-19. J Med Virol. 2020;92(10):2067-73.

41 Schrag D, Hershman DL, Basch E. Oncology practice during the COVID-19 pandemic. JAMA. 2020;323(20):2005-6.

42 Jazieh AR, Chan SL, Curigliano G, Dickson N, Eaton V, Garcia-Foncillas J, et al. Delivering cancer care during the COVID-19 pandemic: recommendations and lessons learned from ASCO global webinars. J Clin Oocol. 2020;6: 1461-71.

43 Raymond E, Thieblemont C, Alran S, Faivre S. Impact of the COVID-19 outbreak on the management of patients with cancer. Target Oncol. 2020;15(3):249-59.

44 Lara OD, O'Cearbhaill RE, Smith MJ, Sutter ME, Knisely A, McEachron J, et al. COVID-19 outcomes of patients with gynecologic cancer in New York city. Cancer. 2020;126(19): 4294-303.
45 Curigliano G, Banerjee S, Cervantes A, Garassino MC, Garrido P, Girard N, et al. Managing cancer patients during the COVID-19 pandemic: an ESMO multidisciplinary expert consensus. Ann Oncol. 2020;31(10):1320-35.

46 Gambichler T, Reuther J, Scheel CH, Susok L, Kern P, Becker JC. Cancer and immune checkpoint inhibitor treatment in the era of SARS-CoV-2 Infection. Cancers. 2020; 12(11):3383.

47 Cancino RS, Su Z, Mesa R, Tomlinson GE, Wang J. The impact of COVID-19 on cancer screening: challenges and opportunities. JMIR Cancer. 2020;6(2):e21697.

48 London JW, Fazio-Eynullayeva E, Palchuk $\mathrm{MB}$, Sankey P, McNair C. Effects of the COVID-19 pandemic on cancer-related patient encounters. JCO Clin Cancer Inform. 2020;4: 657-65.

49 Patt D, Gordan L, Diaz M, Okon T, Grady L, Harmison M, et al. Impact of COVID-19 on cancer care: how the pandemic is delaying cancer diagnosis and treatment for American seniors. JCO Clin Cancer Inform. 2020;4: 1059-71.

50 Maringe C, Spicer J, Morris M, Purushotham A, Nolte E, Sullivan R, et al. The impact of the COVID-19 pandemic on cancer deaths due to delays in diagnosis in England, UK: a national, population-based, modelling study. Lancet Oncol. 2020;21(8):1023-34.

51 Giesen N, Sprute R, Rüthrich M, Khodamoradi Y, Mellinghoff SC, Beutel G, et al. 2021 update of the AGIHO guideline on evidencebased management of COVID-19 in patients with cancer regarding diagnostics, viral shedding, vaccination and therapy. Eur J Cancer. 2021;147:154-60.

52 Mislang AR, Soto-Perez-de-Celis E, Russo C, Colloca G, Williams GR, O'Hanlon S, et al. The SIOG COVID-19 working group recommendations on the rollout of COVID-19 vaccines among older adults with cancer. J Geriatr Oncol. 2021.

53 Baker D, Roberts CAK, Pryce G, Kang AS, Marta M, Reyes S, et al. COVID-19 vaccinereadiness for anti-CD20-depleting therapy in autoimmune diseases. Clin Exp Immunol. 2020;202(2):149-61.

54 Houot R, Levy R, Cartron G, Armand P. Could anti-CD20 therapy jeopardise the efficacy of a SARS-CoV-2 vaccine? Eur J Cancer. 2020;136:4-6.

55 National Institutes of Health. COVID-19 treatment guidelines. Clinical spectrum of SARS-CoV-2 infection; 2021 May 10. Available from: https://www.covid19treatmentguidelines.nih.gov/overview/clinical-spectrum 